\title{
Silver Mesh Electrodes via Electroless-Deposition Coupled Inkjet-Printing Mask Technology for Flexible Polymer Solar Cells
}

\author{
Xiangchuan Meng ${ }^{a, b}$, Qingxia Wang ${ }^{a, b}$, Xia Yang ${ }^{a}$, Jinmao Guo ${ }^{a}$, Xiaotian $\mathrm{Hu}^{b}$, \\ Licheng Tan $* a, b$, Yiwang Chen*a,b \\ ${ }^{a}$ College of Chemistry, Nanchang University, 999 Xuefu Avenue, Nanchang 330031, \\ China \\ ${ }^{b}$ Institute of Polymers and Energy Chemistry (IPEC), Nanchang University, \\ Nanchang 330031, China \\ Corresponding author: Tel.: +86 791 83968703; fax: +86 791 83969561. E-mail: \\ ywchen@ncu.edu.cn (Y.Chen); tanlicheng@ncu.edu.cn (L. Tan).
}

\section{Experimental Section}

Materials. PBDB-T was purchased from 1-Material Chemscitech Inc. and IT-M was purchased from SunaTech Inc. Polystyrene (PS) with molecular weight of 350000 $\mathrm{g} / \mathrm{mol}$, dopamine hydrochloride $\left(\mathrm{C}_{8} \mathrm{H}_{11} \mathrm{NO}_{2} \cdot \mathrm{HCl}, 98 \%\right)$ and methanol $\left(\mathrm{CH}_{3} \mathrm{OH}\right.$, superdry, 99.9\%) were purchased from J\&K Scientific. Ammonium hydroxide solution $\left(\mathrm{NH}_{4} \mathrm{OH}, 28 \% \mathrm{NH}_{3}\right.$ in $\left.\mathrm{H}_{2} \mathrm{O}\right)$, potassium hydroxide $(\mathrm{KOH}, 99.99 \%)$, D-glucose (GC, 99.5\%), chlorobenzene (CB) was received from Sigma-Aldrich. Silver nitrate $\left(\mathrm{AgNO}_{3}, 99.5 \%\right)$ was purchased from Aladdin. Dimethylformamide (DMF, 99.8\%) was obtained from Tianjin Chemical Reagent Co., Ltd. The commercial Ag grid transparent electrodes were bought from InfinityPV Aps. China. Light-emitting diode (LED) bulb was purchased from Jingcheng Electronic Mall.

Tollens' Reaction and PDA Coating was prepared according to a published protocol in previous work. ${ }^{[1]}$ Tris-HCl buffer was prepared by adding Tris-HCl $(0.09 \mathrm{~g})$ and Tris-base $(0.17 \mathrm{~g})$ to $200 \mathrm{~mL}$ deionized water. The cleaned PET substrate was dipped into a dopamine solution $\left(2 \mathrm{mg} / \mathrm{mL}\right.$ in $10 \mathrm{mM}$ Tris- $\mathrm{HCl}$ buffer, $\mathrm{pH} 8.5,25{ }^{\circ} \mathrm{C}$ ) at room temperature for 10-30 $\mathrm{min}$. The dopamine is oxidatively self-polymerized to polydopamine at $\mathrm{pH}=8.5$ condition and deposited on the substrate, following rinsing 
1 with water and $\mathrm{N}_{2}$ drying. According to previous reports of similar work (ACS Appl.

2 Mater. Interfaces, 2014, 6 (3), 1447-1453), the $\mathrm{C}=\mathrm{O}$ peak in XPS spectrum is

3 associated with the substrate PET, suggesting that the thickness of the deposited PDA

4 is below the probing depth of the XPS technique, thus the PDA layer about $10 \mathrm{~nm}$ for

5 organic matrix (Organic Electronics, 2018, 61, 296-303).

Printing Conditions: The PS inks were printed by a Fujifilm Dimatix DMP 3100 printer with $10 \mathrm{pL}$ cartridges. The ink was printed without substrate pre-treatment and then allowed to evaporate at room temperature. The thickness of PS is about $46 \mathrm{~nm}$ from AFM cross-section thickness measurement, as shown in Figure S1c.

\section{Fabrication of PSCs:}

We fabricated the flexible PSCs with the device structure of Ag mesh@PDA/PET /ZnO/PBDB-T:IT-M/MoO $/$ /Al. First, FETs were treated with ultraviolet-ozone plasma for $3 \mathrm{~s}$. Second, the ZnO layer was spin-coated (1500 rpm, $40 \mathrm{~s}$ ) on FETs and then annealed at $150^{\circ} \mathrm{C}$ for $50 \mathrm{~min}$. The material PBDB-T:IT-M was dissolved in CB with the total concentration of $20 \mathrm{mg} / \mathrm{mL}$ and stirred at $40{ }^{\circ} \mathrm{C}$ for $5 \mathrm{~h}$. Subsequently, the active layers were spin coated at $3000 \mathrm{rpm}$ and then thermal annealed for 30 minutes in a glove box. Finally, $\mathrm{MoO}_{3}(10 \mathrm{~nm})$ and $\mathrm{Al}(100 \mathrm{~nm})$ layers were deposited by thermal evaporation under a vacuum of $6 \times 10^{-4}$ Torr. The ITO/PET electrode was also applied in PSCs for comparison.

Characterization. All scanning electron microscopy (SEM) images were measured using FEI XL30 Sirion SEM equipped with EDS. XPS spectra were acquired by SSI SProbe XPS spectrometer with Al Ka source. X-ray diffraction (XRD) pattern was collected by a Bruker D8 Advance X-ray diffractometer operating using Ag $\mathrm{K} \alpha$ radiation $(\lambda=1.5406 \AA)$. Atomic force microscopy (AFM) image was measured with a Veeco D-3100 atomic force microscope. The optical transmittance of samples based on air as a baseline was taken by UV-Vis-NIR spectroscopy (Cary 5000 UV-Vis-NIR, Agilent). The sheet resistance $\left(R_{\mathrm{S}}\right)$ of the conductive films were acquired by a Pro- 4 
1 Lucas Lab four-point probe (digital Keithley 4200-SCS semiconductor parametric

2 analyzer) to eliminate contact resistance. Fifteen randomly selected points were

3 measured on sample. Mechanical bending characterization was evaluated by high

4 precision stretching machine. The samples were bended to a mandrel (with radius of 1 ,

52 and $4 \mathrm{~mm}$ ) up to 2000 cycles. To track the performance degradation, $R_{\mathrm{s}}$ was

6 recorded after certain cycles. An adhesion test (3M $\mathrm{M}^{\mathrm{TM}}$ super 20 epoxy film electrical

7 insulation tape, 3M China) was utilized to assess the adhesive strength between

8 electrodes and PET substrate. The current density-voltage $(J-V)$ curves were measured

9 by a Keithley 2400 source meter with Abet Solar Simulator Sun2000, under AM 1.5G

10 light source $\left(100 \mathrm{~mW} \mathrm{~cm}^{-2}\right)$. The effective area was at $1 \mathrm{~cm}^{2}$. 
1 Table S1. Summary of metal mesh FTEs prepared by different deposition methods.

\begin{tabular}{|c|c|c|c|c|c|c|c|c|}
\hline Method & FTES & $\begin{array}{c}\text { Thickness } \\
\text { (nm) }\end{array}$ & $\begin{array}{l}\text { Thickness } \\
\text { after } \\
\text { transfer }\end{array}$ & $\begin{array}{c}\mathrm{T} \\
(\%)\end{array}$ & $\begin{array}{c}R_{\mathrm{s}} \\
(\Omega / \mathrm{sq})\end{array}$ & Application & Mechanical flexibility & Ref. \\
\hline Electrospun+Electroless & $\mathrm{Ag}$ & $70 \mathrm{~nm}$ & - & 87.2 & 22.9 & $\begin{array}{l}6.4 \% \text { of PCE in } \\
\text { PSCs }\end{array}$ & $\begin{array}{c}R_{\mathrm{S}} \text { increasing by } 0.1 \% \\
\text { after } 1000 \text { bending } \\
\text { cycles }\end{array}$ & 1 \\
\hline Lithography+Electroless & $\mathrm{Cu}$ & $945 \mathrm{~nm}$ & - & $>80$ & $<1$ & light emitter & $\begin{array}{c}R_{\mathrm{S}} \text { increasing by } 3 \text { times } \\
\text { after } 1000 \text { bending } \\
\text { cycles }\end{array}$ & 2 \\
\hline Lithography+Electroplating & $\mathrm{Cu}$ & $1000 \mathrm{~nm}$ & - & $>80$ & $<2$ & $\begin{array}{l}\text { light-emitting } \\
\text { film }\end{array}$ & $\begin{array}{c}R_{\mathrm{s}} \text { increasing by } 5 \text { times } \\
\text { after } 500 \text { cycles at } 55 \% \\
\text { stretching }\end{array}$ & 3 \\
\hline Lithography+Electroplating & $\mathrm{Cu}$ & $1800 \mathrm{~nm}$ & $50 \mathrm{~nm}$ & $>90$ & $<1$ & heater & $\begin{array}{c}R_{\mathrm{s}} \text { increasing by } 2 \text { times } \\
\text { after } 1000 \text { bending } \\
\text { cycles }\end{array}$ & 4 \\
\hline Self-cracks+Electroless & $\mathrm{Ag}$ & - & $3000 \mathrm{~nm}$ & 85 & 1 & - & - & 5 \\
\hline Convective self-assembly & $\mathrm{Ag}$ & $270 \mathrm{~nm}$ & $17 \mathrm{~nm}$ & 90 & 5.7 & $\begin{array}{l}3.1 \% \text { of PCE in } \\
\text { PSCs }\end{array}$ & $\begin{array}{c}R_{\mathrm{S}} \text { increasing by } 0.5 \\
\text { times after } 1000 \\
\text { bending cycles }\end{array}$ & 6 \\
\hline Direct-printing+Electroless & $\mathrm{Au}$ & $100 \mathrm{~nm}$ & - & $>90$ & - & transistors & $\begin{array}{l}\text { no degradation of } R_{\mathrm{S}} \\
\text { after } 1000 \text { bending } \\
\text { cycles }\end{array}$ & 7 \\
\hline Chemisorption printing & $\mathrm{Ag}$ & $100 \mathrm{~nm}$ & - & 75 & 4 & touch sensor & - & 8 \\
\hline Soft nanoimprinting & $\mathrm{Ag}$ & $2000 \mathrm{~nm}$ & - & 89.7 & 2 & supercapacitors & $\begin{array}{c}\text { capacitance loss of } \\
2.6 \% \text { after } 1000 \text { cycles }\end{array}$ & 9 \\
\hline Reverse-offset printing & $\mathrm{Ag}$ & - & $100 \mathrm{~nm}$ & 93.2 & 17 & $\begin{array}{l}8.3 \% \text { of PCE in } \\
\text { PSCs }\end{array}$ & $\begin{array}{c}R_{\mathrm{s}} \text { increasing by } 10.6 \% \\
\text { after } 500 \text { cycles at } \\
100 \% \text { strain }\end{array}$ & 10 \\
\hline Inkjet printing+Electroless & $\mathrm{Cu}$ & $705 \mathrm{~nm}$ & - & - & 35.8 & - & $\begin{array}{c}R_{\mathrm{s}} \text { increasing by } 13 \% \\
\text { after } 10000 \text { bending } \\
\text { cycles }\end{array}$ & 11 \\
\hline Inkjet printing+Electroless & $\mathrm{Ag}$ & $400 \mathrm{~nm}$ & - & - & 18 & - & $\begin{array}{c}R_{\mathrm{S}} \text { increase rapidly after } \\
1000 \text { bending cycles }\end{array}$ & 12 \\
\hline Inkjet printing+Electroless & Ag & $50 \mathrm{~nm}$ & - & 89.9 & 9 & $\begin{array}{c}10.24 \% \text { of PCE } \\
\text { in PSCs, > 81\% } \\
\text { of initial PCE } \\
\text { after } 1500 \\
\text { bending cycles }\end{array}$ & $\begin{array}{c}R_{\mathrm{s}} \text { increasing by } 1.15 \\
\text { times after } 2000 \\
\text { bending cycles }\end{array}$ & $\begin{array}{l}\text { this } \\
\text { work }\end{array}$ \\
\hline
\end{tabular}

2 The active layer of Ref. 1, 6, 12 and our work consist of PTB7-Th:PC71BM,

3 PTB7-PC71BM, PTzNTz- PC71BM, and PBDB-T:IT-M, respectively. 
1 Table S2. Photovoltaic performance of the flexible devices based on the commercial

2 Ag girds and Ag mesh@PDA/PET electrodes with different PS matrix thickness.

\begin{tabular}{|c|c|c|c|c|}
\hline FTEs & $\begin{array}{c}J_{\mathrm{sc}} \\
\left.(\mathrm{mA} \mathrm{cm})^{-2}\right)\end{array}$ & $\begin{array}{l}V_{\text {oc }} \\
(V)\end{array}$ & $\begin{array}{l}\text { FF } \\
(\%)\end{array}$ & $\begin{array}{l}\text { PCE } \\
(\%)\end{array}$ \\
\hline \multirow{2}{*}{ Commercial Ag grids } & $7.04^{\mathrm{a}}$ & $0.345^{\mathrm{a}}$ & $30.2^{\mathrm{a}}$ & $0.734^{\mathrm{a}}$ \\
\hline & $5.89 \pm 1.26$ & $0.205 \pm 0.140$ & $24.6 \pm 6.4$ & $0.32 \pm 0.30$ \\
\hline \multirow{2}{*}{ PS matrix (46 nm) } & $15.04^{\mathrm{a}}$ & $0.950^{\mathrm{a}}$ & $71.7^{\mathrm{a}}$ & $10.24^{\mathrm{a}}$ \\
\hline & $15.04 \pm 1.26$ & $0.950 \pm 0.020$ & $70.7 \pm 2.4$ & $9.73 \pm 0.51$ \\
\hline \multirow{2}{*}{ PS matrix (90 nm) } & $12.56^{\mathrm{a}}$ & $0.947^{\mathrm{a}}$ & $62.5^{\mathrm{a}}$ & $7.43^{\mathrm{a}}$ \\
\hline & $11.23 \pm 1.56$ & $0.945 \pm 0.020$ & $60.5 \pm 1.9$ & $6.42 \pm 0.98$ \\
\hline \multirow{2}{*}{ PS matrix (136 nm) } & $10.75^{\mathrm{a}}$ & $0.910^{\mathrm{a}}$ & $55.8^{\mathrm{a}}$ & $5.46^{\mathrm{a}}$ \\
\hline & $9.26 \pm 1.45$ & $0.890 \pm 0.030$ & $50.5 \pm 5.5$ & $4.16 \pm 1.35$ \\
\hline
\end{tabular}

3 The statistical results are obtained from ten devices. The effective area is $1 \mathrm{~cm}^{2}$.

4 The flexible device is based on PET/FTEs/ZnO/PBDB-T:IT-M/MoO $/$ /Al.

5 a The best $J_{\mathrm{sc}}, V_{\mathrm{oc}}, \mathrm{FF}$ and PCE values. 
(a)

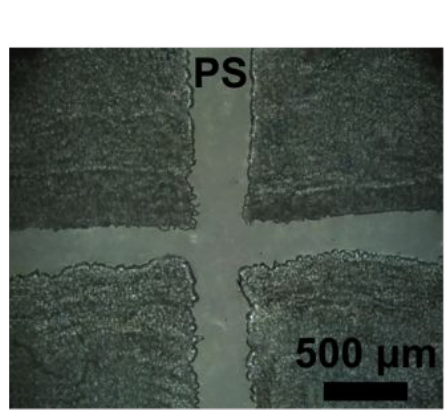

(b)

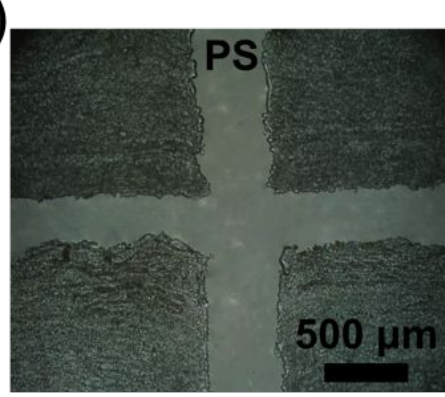

(c)

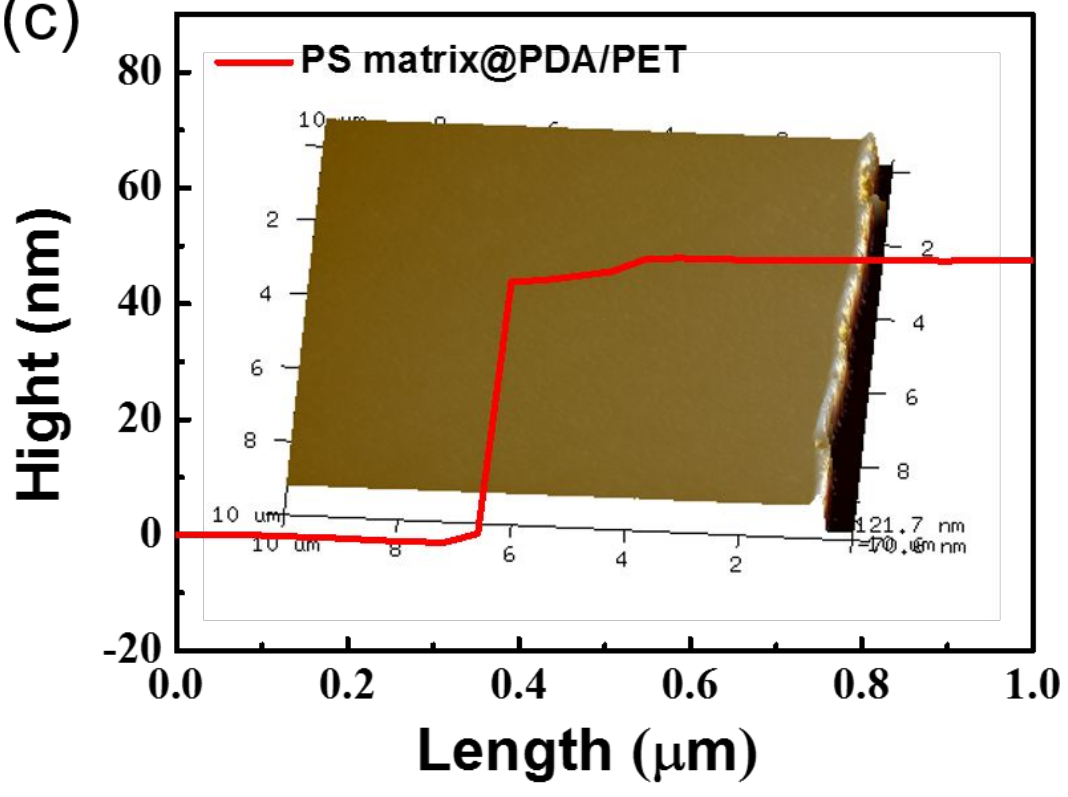

2 Figure S1. Optical microscope images of printed PS matrices with clear edge. The

3 pitches of the printed PS matrices are (a) $300 \mu \mathrm{m}$ and (b) $500 \mu \mathrm{m}$. (c) The thickness

4 difference between PS matrix and PET substrate (the inset is the 3-D image of AFM 5 cross-section measure). 
(a)

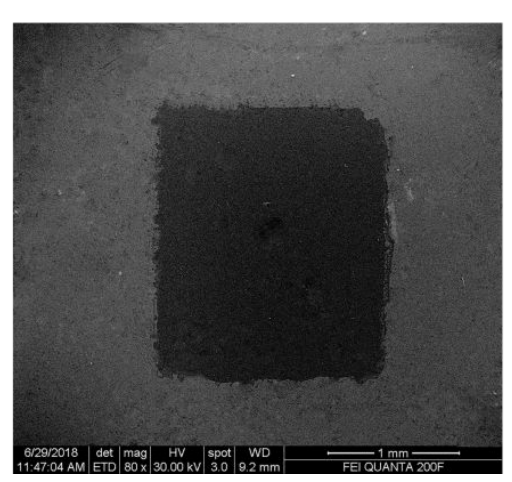

(b)

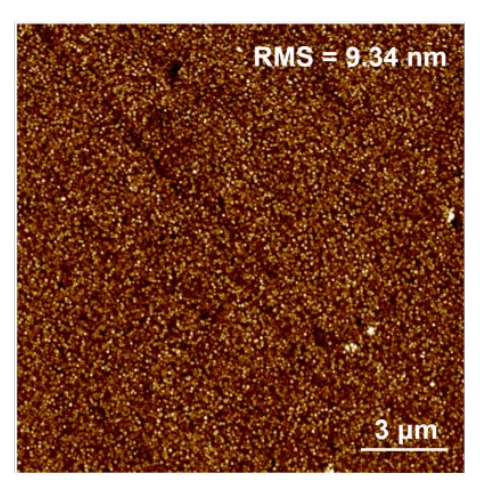

1

2 Figure S2. (a) SEM and (b) AFM images of Ag mesh@PDA/PET. 
(a)

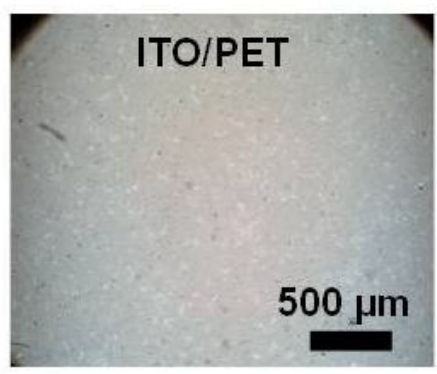

(b)

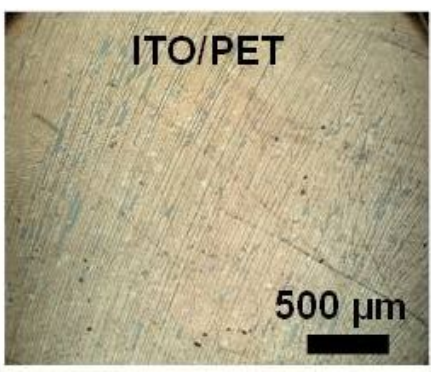

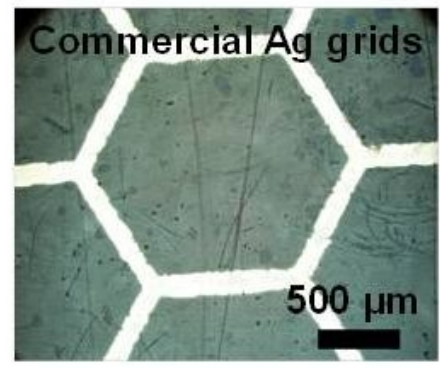

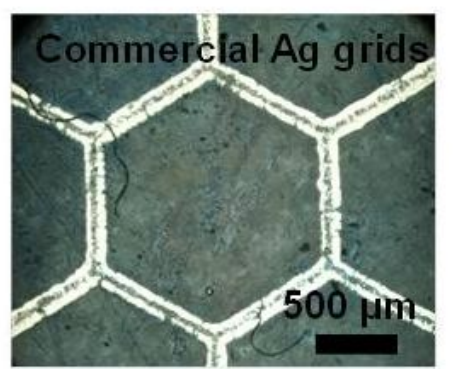

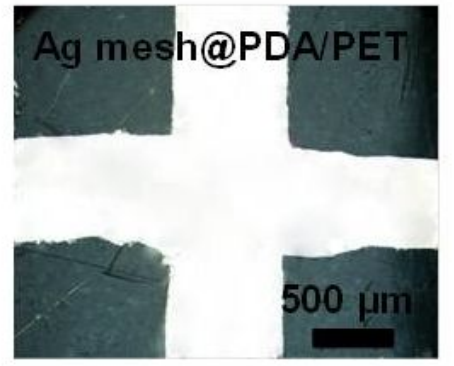

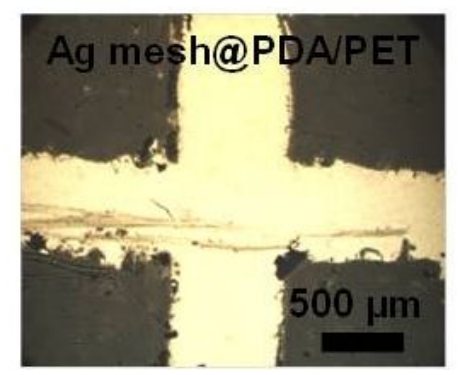

2 Figure S3. Optical microscope images of FTEs (a) before and (b) after different 3 bending cycles with bending radius of $1 \mathrm{~mm}$ : only 100 bending cycles (ITO/PET),

42000 bending cycles (commercial Ag grids), and 2000 bending cycles (Ag 5 mesh@PDA/PET), respectively. 


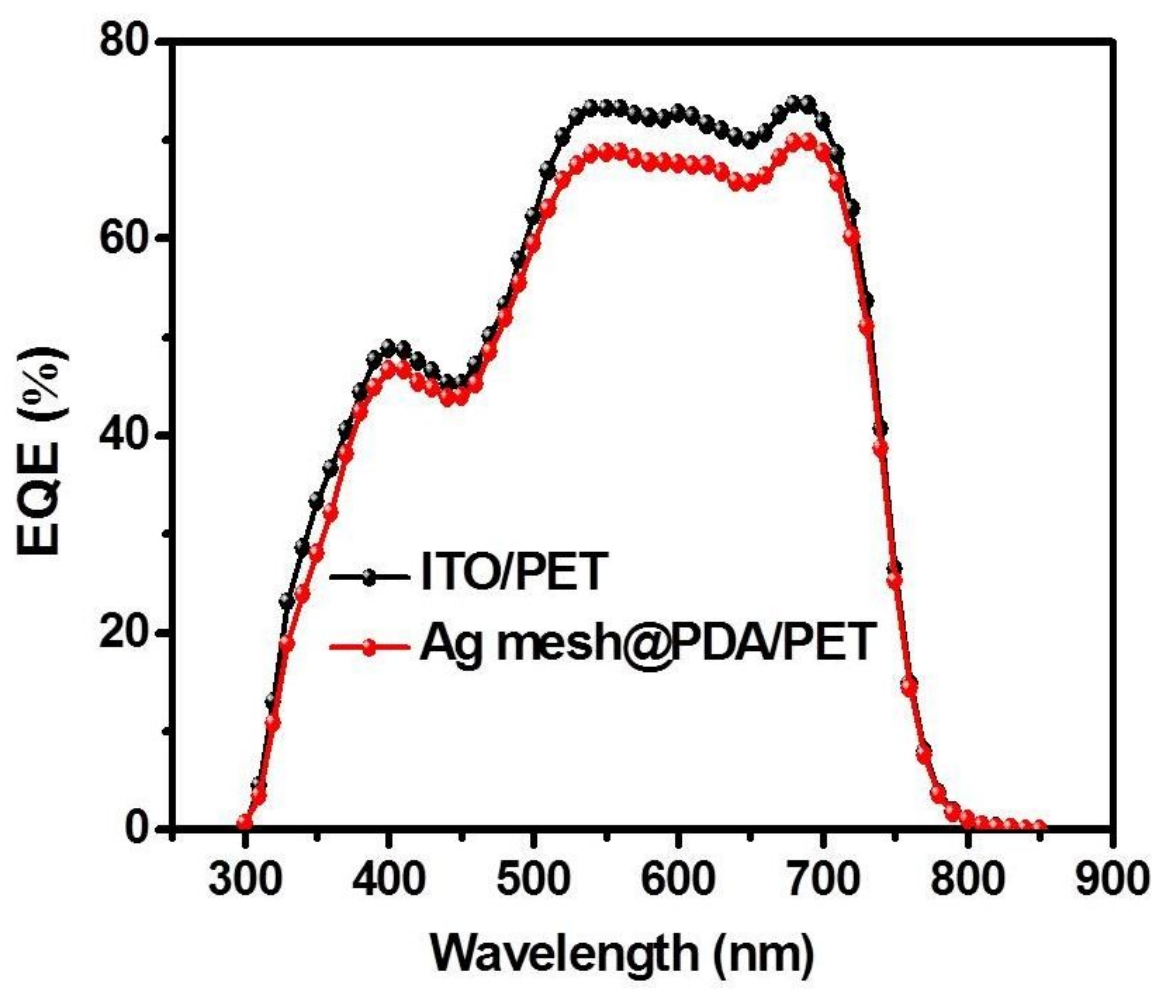

2 Figure S4. EQE spectra of flexible PSCs based on Ag mesh@PDA/PET and 3 ITO/PET transparent electrodes. 


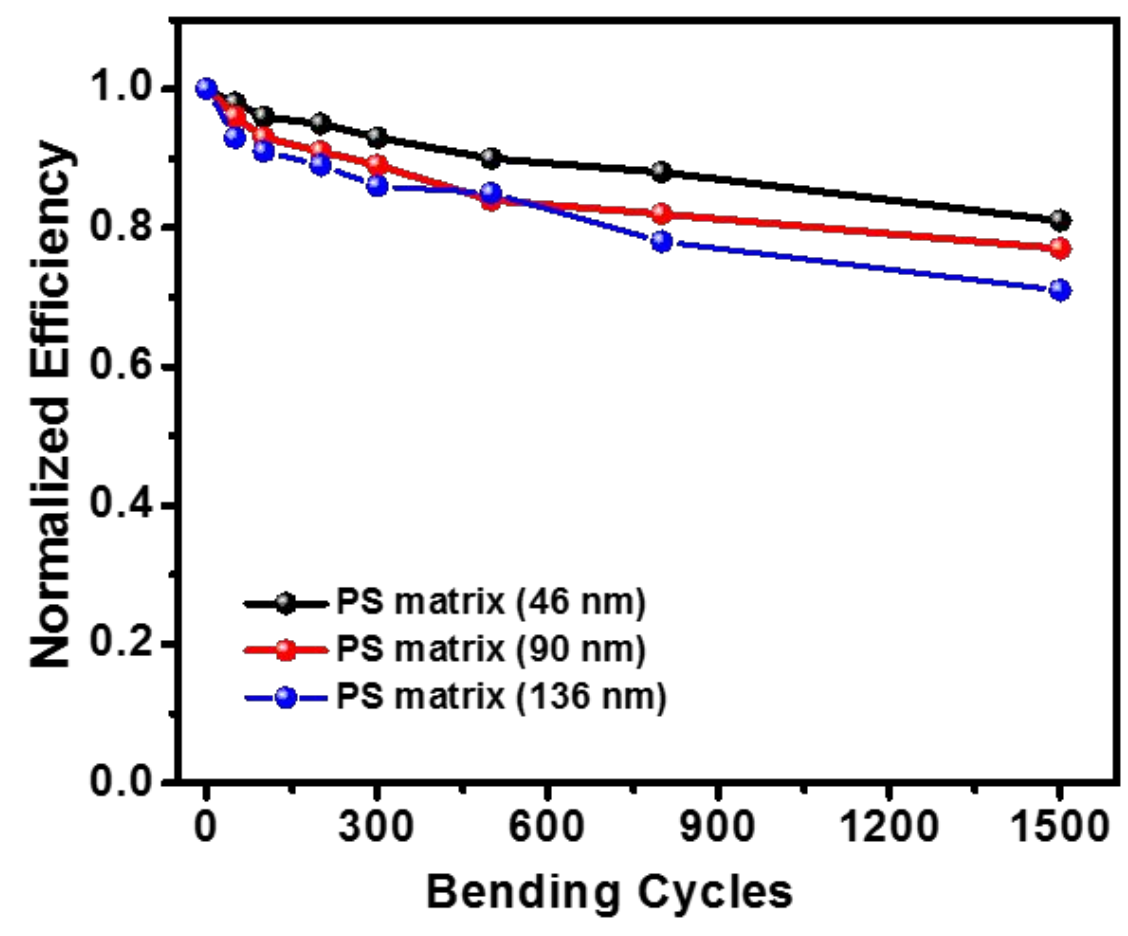

2 Figure S5. Normalized average power conversion efficiency (PCE) of flexible PSCs

3 without encapsulation as a function of bending cycles (radius of $4 \mathrm{~mm}$ ) with different $4 \quad$ PS matrix thickness. 
1 Video S1. The brightness change of LED lamp on different location of the electric

2 circuit under mechanically bending with radius of $4 \mathrm{~mm}$. 


\section{References}

[1] Wang, Q., Hu, X., Yang, X., Liu, G., Meng, X., Xie, Y., Xiao, Y., Liu, J., Tan, L., Chen, Y. Large-Scale Ultra-Adhesive and Mechanically Flexible Silver Grids Transparent Electrodes by Solution Process. Org. Electron. 2018, 61, 296-303.

[2] Cai, J., Zhang, C., Khan, A., Wang, L., Li, W. Selective Electroless Metallization of Micro- and Nanopatterns via Poly(dopamine) Modification and Palladium Nanoparticle Catalysis for Flexible and Stretchable Electronic Applications. ACS Appl. Mater. Interfaces 2018, 10(34), 28754-28763.

[3] Zhang, C., Khan, A., Cai, J., Liang, C., Liu, Y., Deng, J., Huang, S., Li, G., Li, W. Stretchable Transparent Electrodes with Solution-Processed Regular Metal Mesh for an Electroluminescent Light-Emitting Film. ACS Appl. Mater. Interfaces 2018, 10(24), 21009-21017.

[4] Khan, A., Lee, S., Jang, T., Xiong, Z., Zhang, C., Tang, J., Guo, L., Li, W. High-Performance Flexible Transparent Electrode with an Embedded Metal Mesh Fabricated by Cost-Effective Solution Process. Small 2016, 12(22), 3021-3030.

[5] Xian, Z., Han, B., Li, S., Yang, C., Wu, S., Lu, X., Gao, X., Zeng, M., Wang, Q., Bai, P., Naughton, M., Zhou, C., Liu, J., Kempa, K., Gao, J. A Practical ITO Replacement Strategy: Sputtering-Free Processing of a Metallic Nanonetwork. Adv. Mater. Technol. 2017, 2(8), 1700061

[6] Choi, D. Y., Oh, Y. S., Han, D., Yoo, S., Sung, H. J., Kim, S. S. Highly Conductive, Bendable, Embedded Ag Nanoparticle Wire Arrays via Convective Self-assembly: Hybridization into Ag Nanowire Transparent Conductors. Adv. Funct. Mater. 2015, 25(25), 3888-3898.

[7] Min, S. Y., Lee, Y. J., Kim, S. H., Park, T. C., Lee, W. Room-Temperature-Processable Wire-Templated Nanoelectrodes for Flexible and Transparent All-Wire Electronics. ACS Nano 2017, 11(4), 3681-3689.

[8] Yamada, T., Fukuhara, K., Matsuoka, K., Minemawari, H., Tsutsumi, J., Fukuda, N., Aoshima, K., Arai, S., Makita, Y., Kubo, H., Enomoto, T., Togashi, T., Kurihara, M., Hasegawa, T. Nanoparticle Chemisorption Printing Technique for Conductive Silver Patterning with Submicron Resolution. Nat. Commun. 2016, 7, 
11402.

2 [9] Xu, J., Liu, Y., Gao, X., Sun, Y., Shen, S., Cai, X., Chen, L., Wang, S. Embedded Ag Grid Electrodes as Current Collector for Ultraflexible Transparent Solid-State Supercapacitor. ACS Appl. Mater. Interfaces 2017, 9(33), 27649-27656.

[10] Jiang, Z., Fukuda, K., Xu, X., Park, S., Inoue, D., Jin, H., Saito, M., Osaka, I.,

Conformal Transparent Electrodes for High-Performance Organic Photovoltaics. Adv. Mater. 2018, 30(26), 1707526.

[11] Ma, S., Liu, L., Bromberg, V., Singler, T. J. Electroless Copper Plating of Inkjet-Printed Polydopamine Nanoparticles: a Facile Method to Fabricate Highly 1 Conductive Patterns at Near Room Temperature. ACS Appl. Mater. Interfaces 2 2014, 6(22), 19494-19498.

[12] Ma, S., Liu, L., Bromberg, V., Singler, T. J. Fabrication of Highly Electrically 4 Conducting Fine Patterns via Substrate-Independent Inkjet Printing of 5 Mussel-Inspired Organic Nano-Material. J. Mater. Chem. C 2014, 2(20), $16 \quad 3885-3889$. 